\title{
Stereoselective and Efficient Total Synthesis of Optically Active Tetrodotoxin from D-Glucose
}

Ken-ichi Sato, * Shoji Akai, Hirotsugu Shoji, Naoki Sugita, Shiho Yoshida, Yoshinao Nagai, Katsuhiko Suzuki, Yutaka Nakamura, Yasuhiro Kajihara,

Masuo Funabashi, ${ }^{1}$ and Juji Yoshimura ${ }^{2}$

Laboratory of Organic Chemistry, Faculty of Engineering, Kanagawa University,

3-27-1 Rokkakubashi, Kanagawa-ku, Yokohama, 221-8686, Japan

${ }^{1}$ Department of Chemistry, Faculty of Sciences, Chiba University,

1-33 Yayoi-cho, Inage-ku, Chiba-shi, 263-8522, Japan

${ }^{2}$ Department of Basic Science, College of Science and Engineering, Iwaki Meisei University, 5-5-1 Iino, Chuodai, Iwaki, 970-8551, Japan

\section{Supporting Information}

Table of contents.

Experimental

S1-S14

Figure

Spectra

S16-S54 


\section{Experimental}

\section{General Methods}

All of the melting points are uncorrected. The optical rotations were measured in a $0.5 \mathrm{dm}$ tube in $\mathrm{CHCl}_{3}$.

${ }^{1} \mathrm{H}$ NMR spectra were recorded on spectrometers operating at $200 \mathrm{MHz}, 500 \mathrm{MHz}$, and $600 \mathrm{MHz}$ in chloroform- $d$ referenced to tetramethylsilane $(0.00 \mathrm{ppm}) .{ }^{13} \mathrm{C}$ NMR spectra were recorded on spectrometers operating at $125 \mathrm{MHz}$ and $150 \mathrm{MHz}$ in chloroform- $d$. The assignments of various NMR spectra were assisted by homonuclear $\left({ }^{1} \mathrm{H} /{ }^{1} \mathrm{H}\right)$ correlation spectroscopy (COSY), nuclear Overhuser and exchange spectroscopy (NOESY), nuclear Overhauser effect (NOE), and/or heteronuclear $\left({ }^{1} \mathrm{H} /{ }^{13} \mathrm{C}\right)$ correlation spectroscopy (HETCOR) experiments. The chemical shifts, coupling constants, and IR frequencies were recorded in $\delta, \mathrm{Hz}$, and $\mathrm{cm}^{-1}$ units, respectively. Column chromatography was performed on silica gel (Silica gel 60, 70-230 mesh; Silica gel 60N, spherical, neutral, 70-230 mesh). Thin-layer chromatography (TLC) on (Silica gel $60 \mathrm{~F}_{254}$ ) was used to monitor the reactions and to certify the purity of the reaction products by charring after spraying with $5 \% \mathrm{H}_{2} \mathrm{SO}_{4}$ in ethanol. X-ray diffraction was measurement with graphite monochromated Mo-K $\alpha$ radiation.

3-Deoxy-1,2:5,6-Di-O-isopripylidene-3- $C$-methylene- $\alpha$-D-ribo-hexofuranose (2). This compound was prepared by the reported methods in $66 \%$ yield from D-glucose $;^{22}$ colorless syrup; $[\alpha]_{\mathrm{D}}^{24}+107.4^{\circ}(c 1.0$, $\left.\mathrm{CHCl}_{3}\right)$; IR (KBr, neat) v $1680 \mathrm{~cm}^{-1}(\mathrm{C}=\mathrm{C}) ; \quad \mathrm{H}$ NMR $(600 \mathrm{MHz}) \delta 5.81\left(1 \mathrm{H}, \mathrm{d}, J_{1,2}=4.1 \mathrm{~Hz}, \mathrm{H}-1\right), 5.51$ $\left(1 \mathrm{H}, \mathrm{dd}, J_{2,3^{\prime} \mathrm{a}}=1.0 \mathrm{~Hz}, J_{4,3^{\prime} \mathrm{a}}=2.2 \mathrm{~Hz}, \mathrm{H}-3{ }^{\prime} \mathrm{a}\right), 5.44\left(1 \mathrm{H}, \mathrm{dd}, J_{2,3^{\prime} \mathrm{b}}=1.0 \mathrm{~Hz}, J_{4,3^{\prime} \mathrm{b}}=2.2 \mathrm{~Hz}, \mathrm{H}-3^{\prime} \mathrm{b}\right), 4.89$ 
$(1 \mathrm{H}, \mathrm{ddd}, \mathrm{H}-2), 4.65\left(1 \mathrm{H}, \mathrm{ddd}, J_{4,5}=5.5 \mathrm{~Hz}, \mathrm{H}-4\right), 4.07\left(1 \mathrm{H}, \mathrm{dd}, J_{6,5}=8.2 \mathrm{~Hz}, J_{6,6}=6.4 \mathrm{~Hz}, \mathrm{H}-6\right), 4.06$

$\left(1 \mathrm{H}, \mathrm{dd}, J_{5,6^{\prime}}=4.5 \mathrm{~Hz}, \mathrm{H}-6\right.$ ') $3.94(1 \mathrm{H}, \mathrm{ddd}, \mathrm{H}-5), 1.52,1.43,1.37,1.36\left(12 \mathrm{H}, 4 \times \mathrm{s}, 2 \times \mathrm{C}\left(\mathrm{CH}_{3}\right)_{2}\right)$.

3,3'-Anhydro-3-C-hydroxymethyl-1,2:5,6-di- $O$-isopropylidene- $\alpha$-D-glucofuranose

(3). This

compound was prepared by a reference method in $86 \%$ yield $;^{21}$ colorless syrup; $[\alpha]_{\mathrm{D}}^{24}+53.6^{\circ}(c 1.1$, $\left.\mathrm{CHCl}_{3}\right) ;{ }^{1} \mathrm{H}$ NMR $(600 \mathrm{MHz}) \delta 5.95\left(1 \mathrm{H}, \mathrm{d}, J_{1,2}=4.0 \mathrm{~Hz}, \mathrm{H}-1\right), 4.37\left(1 \mathrm{H}, \mathrm{d}, J_{4,5}=7.0 \mathrm{~Hz}, \mathrm{H}-4\right), 4.29(1 \mathrm{H}$, d, H-2), 4.08-4.00 (3H, m, H-5, H-6, H-6'), 3.16, 3.09 (2H, $2 \times$ d, $J_{\mathrm{a}, \mathrm{b}}=4.9 \mathrm{~Hz}, \mathrm{H}-3$ ') $, 1.56,1.40,1.34$, $1.31\left(12 \mathrm{H}, 4 \times \mathrm{s}, 2 \times \mathrm{C}\left(\mathrm{CH}_{3}\right)_{2}\right)$.

3- $C$-Hydroxymethyl-1,2;5,6-di- $O$-isopropylidene- $\alpha$-D-glucofuranose (4). Alcohol 4 was prepared by a reference method in $86 \%$ yield ${ }^{[21]} \mathrm{mp} 85.0-86.0{ }^{\circ} \mathrm{C}$ (colorless needles, ether-hexane); $[\alpha]_{\mathrm{D}}^{24}+25.6^{\circ}(c$ 0.98, $\left.\mathrm{CHCl}_{3}\right)$; IR (KBr, neat) v $3400 \mathrm{~cm}^{-1}(\mathrm{OH}) ; \quad \mathrm{H}$ NMR $(600 \mathrm{MHz}) \delta 5.87\left(1 \mathrm{H}, \mathrm{d}, J_{1,2}=3.6 \mathrm{~Hz}, \mathrm{H}-1\right)$, $4.40(1 \mathrm{H}, \mathrm{d}, \mathrm{H}-2), 4.27\left(1 \mathrm{H}, \mathrm{ddd}, J_{5,4}=8.7 \mathrm{~Hz}, J_{5,6}=4.9 \mathrm{~Hz}, J_{5,6}=6.3 \mathrm{~Hz}, \mathrm{H}-5\right), 4.17\left(1 \mathrm{H}, \mathrm{dd}, J_{6,6}=8.9\right.$ Hz, H-6), 4.06 (1H, dd, H-6’ $), 3.86\left(1 \mathrm{H}, \mathrm{dd}, J_{3^{\prime} \mathrm{a}, \mathrm{OH}}=3.4 \mathrm{~Hz}, J_{3^{\prime} \mathrm{a}, 3^{\prime} \mathrm{b}}=12.0 \mathrm{~Hz}, \mathrm{H}-3^{\prime}{ }^{\prime} \mathrm{a}\right), 3.83(1 \mathrm{H}, \mathrm{d}, \mathrm{H}-4)$, $3.81\left(1 \mathrm{H}, \mathrm{dd}, J_{3}{ }^{\prime} \mathrm{b}, \mathrm{OH}=10.6 \mathrm{~Hz}, \mathrm{H}^{\prime} 3^{\prime} \mathrm{b}\right), 3.26(1 \mathrm{H}, \mathrm{s}, \mathrm{OH}), 3.19\left(1 \mathrm{H}, \mathrm{dd}, \mathrm{CH}_{2} \mathrm{OH}\right), 1.51,1.45,1.37,1.31$ $\left(12 \mathrm{H}, 4 \times \mathrm{s}, 2 \times \mathrm{C}\left(\mathrm{CH}_{3}\right)_{2}\right) ; \quad{ }^{3} \mathrm{C} \mathrm{NMR}(150 \mathrm{MHz}) \delta 112.7,109.8,104.6,86.6,82.9,80.7,71.8,67.7,62.4$, $26.9,26.7,26.2,25.1$.

1D-(1,2,3,5,6/4(OH))-6'-O-tert-butyldiphenylsilyl-1'-C-dichloromethyl-4,6-di-C-hydroxymethyl-2,3:4 ,4'-di- $O$-isopropylidene-5- $O$-methoxymethyl-1,2,3,4,5-cyclohexanepentol (20). To a stirred solution of $N, N$-diisopropylamine $(0.599 \mathrm{~mL}, 4.26 \mathrm{mmol})$ in THF $(40 \mathrm{~mL})$, butyllithium $(1.54 \mathrm{M}$ solution in hexane, 
$2.77 \mathrm{~mL}, 4.27 \mathrm{mmol})$ was added dropwise at $-78{ }^{\circ} \mathrm{C}$ under argon. After $30 \mathrm{~min}$, dry $\mathrm{CH}_{2} \mathrm{Cl}_{2}(0.91 \mathrm{~mL}$, $14.2 \mathrm{mmol}$ ) was added dropwise, and then a solution of 19 (389 $\mathrm{mg}, 0.665 \mathrm{mmol})$ in THF (3 mL) was added dropwise, and stirred at $-78{ }^{\circ} \mathrm{C}$ under argon. After the disappearance of 19 on TLC with 4:1 hexane-EtOAc, the reaction mixtures was poured into aq $\mathrm{NH}_{4} \mathrm{Cl}$ solution, extracted with $\mathrm{CHCl}_{3}$, washed, brine and water, dried over anhyd $\mathrm{MgSO}_{4}$, and evaporated to give 20 (355 mg, 79\% yield), which was purified on a column of silica gel with 8:1 hexane-EtOAc; mp 98.0-99.0 ${ }^{\circ} \mathrm{C}$ (colorless needles, hexane-EtOH); $[\alpha]_{\mathrm{D}}^{26}-24.8^{\circ}\left(c 0.91, \mathrm{CHCl}_{3}\right) ; \mathrm{IR}(\mathrm{KBr}$, disk $) \vee 3464 \mathrm{~cm}^{-1}(\mathrm{OH}) ; \mathrm{H} \mathrm{NMR}(600 \mathrm{MHz}) \delta$ 7.68-7.36 (10H, m, PhH), $5.70\left(1 \mathrm{H}, \mathrm{s}, \mathrm{H}-1^{\prime}\right), 4.83,4.68\left(2 \mathrm{H}, 2 \times \mathrm{d}, J_{\mathrm{A}, \mathrm{B}}=6.9 \mathrm{~Hz}, \mathrm{CH}_{2} \mathrm{OCH}_{3}\right), 4.50,(1 \mathrm{H}$, s, OH), $4.44\left(1 \mathrm{H}, \mathrm{d}, J_{2,3}=6.9 \mathrm{~Hz}, \mathrm{H}-2\right), 4.36,4.32\left(2 \mathrm{H}, 2 \times \mathrm{d}, J_{\mathrm{a}, \mathrm{b}}=9.6 \mathrm{~Hz}, \mathrm{H}-4^{\prime}\right), 4.34\left(1 \mathrm{H}, \mathrm{br} \mathrm{d}, J_{5,6}=\right.$

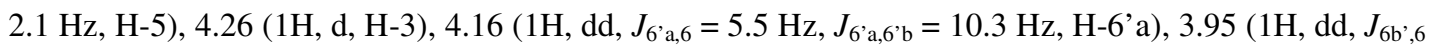
$=9.6 \mathrm{~Hz}, \mathrm{H}-6$ 'b), $3.35\left(3 \mathrm{H}, \mathrm{s}, \mathrm{OCH}_{3}\right), 2.63(1 \mathrm{H}, \mathrm{ddd}, \mathrm{H}-6), 1.52,1.46,1.42,1.39(12 \mathrm{H}, 4 \times \mathrm{s}, 2 \times$ $\left.\mathrm{C}\left(\mathrm{CH}_{3}\right)_{2}\right), 1.06\left(9 \mathrm{H}, \mathrm{s}, \mathrm{C}\left(\mathrm{CH}_{3}\right)_{3}\right)$. Anal. Calcd. for $\mathrm{C}_{33} \mathrm{H}_{46} \mathrm{O}_{8} \mathrm{Si}$ (669.70): C, 59.12; H, 6.92. Found: $\mathrm{C}$, $58.90 ; \mathrm{H}, 6.88$.

1D-(1(OH),2,3,5,6/4(OH))-1,1'-Anhydro-6'-O-tert-butyldiphenylsilyl-2,3:4,4'-di- $O$-isopropylidene-5-

$O$-methoxymethyl-1- $C$-(1'-chloro-1'-hydroxymethyl)-4,6-di- $C$-hydroxymethylcyclohexane-1,2,3,4,5-

pentol (21). colorless syrup; ${ }^{1} \mathrm{H}$ NMR (600 MHz) $\delta 7.67-7.61,7.43-7.32(10 \mathrm{H}, 2 \times \mathrm{m}, 2 \times \mathrm{Ph} H), 5.06$, $4.65\left(2 \mathrm{H}, 2 \times \mathrm{d}, J_{\mathrm{A}, \mathrm{B}}=7.0 \mathrm{~Hz}, \mathrm{CH}_{2} \mathrm{OCH}_{3}\right), 4.91\left(1 \mathrm{H}, \mathrm{s}, \mathrm{H}-1^{\prime}\right), 4.40,4.23\left(2 \mathrm{H}, 2 \times \mathrm{d}, J_{\mathrm{a}, \mathrm{b}}=9.5 \mathrm{~Hz}, \mathrm{H}-4\right.$ '), $4.22\left(1 \mathrm{H}, \mathrm{dd}, J_{6^{\prime} \mathrm{a}, 6}=10.0 \mathrm{~Hz}, J_{6^{\prime} \mathrm{a}, 6^{\prime} \mathrm{b}}=10.0 \mathrm{~Hz}, \mathrm{H}-6^{\prime} \mathrm{a}\right), 4.19\left(1 \mathrm{H}, \mathrm{d}, J_{5,6}=6.7 \mathrm{~Hz}, \mathrm{H}-5\right), 4.14\left(1 \mathrm{H}, \mathrm{dd}, J_{5,3}\right.$ 
$=1.7 \mathrm{~Hz}, \mathrm{H}-3), 3.78\left(1 \mathrm{H}, \mathrm{dd}, J_{6^{\prime} \mathrm{b}, 6}=4.0 \mathrm{~Hz}, \mathrm{H}-6\right.$ ’ b), $3.75\left(1 \mathrm{H}, \mathrm{d}, J_{2,3}=7.7 \mathrm{~Hz}, \mathrm{H}-3\right), 3.40(3 \mathrm{H}, \mathrm{s}$, $\left.\mathrm{CH}_{2} \mathrm{OCH}_{3}\right), 2.74(1 \mathrm{H}, \mathrm{ddd}, \mathrm{H}-6), 1.51,1.43,1.38,1.23\left(12 \mathrm{H}, 4 \times \mathrm{s}, 2 \times \mathrm{C}\left(\mathrm{CH}_{3}\right)_{2}\right), 1.04(\mathrm{~s}, 9 \mathrm{H}$, $\left.\mathrm{SiC}\left(\mathrm{CH}_{3}\right)_{3}\right)$.

\section{D-(1,2,3,5,6/4(OH))-1'-Azido-6'-O-tert-butyldiphenylsilyl-4,6-di- $C$-hydroxymethyl-2,3:4,4'-di-O-iso}

propylidene-5- $O$-methoxymethyl-2,3,4,5-tetrahydroxycyclohexanecarbaldehyde (22). To a solution of dichloroethanol derivative $20(16.6 \mathrm{mg}, 248 \mu \mathrm{mol})$ in dry dimethylsulfoxide $(0.4 \mathrm{~mL})$, sodium azide (10.0 mg, $149 \mu \mathrm{mol})$ and 15-crown-5 ether $(15 \mu \mathrm{L}, 742 \mu \mathrm{mol})$ were added and stirred at $90{ }^{\circ} \mathrm{C}$ under argon for $14 \mathrm{~h}$. After the disappearance of starting compound on TLC with 6:1 hexane-EtOAc, the reaction mixture was poured into aq $\mathrm{NH}_{4} \mathrm{Cl}$, extracted with EtOAc, washed, brine and water, dried over anhyd $\mathrm{MgSO}_{4}$, and evaporated to give $22(11.8 \mathrm{mg}, 74 \%$ yield $)$, which was purified on a column of silica gel with 6:1 hexane-EtOAc; mp 108.0-109.0 ${ }^{\circ} \mathrm{C}$ (colorless prisms, hexane-EtOH); $[\alpha]_{\mathrm{D}}^{26}-62.7^{\circ}(c 1.07$, $\left.\mathrm{CHCl}_{3}\right)$; IR (KBr, disk) v $2116 \mathrm{~cm}^{-1}\left(\mathrm{~N}_{3}\right), 1724 \mathrm{~cm}^{-1}(\mathrm{C}=\mathrm{O}) ; \quad \mathrm{H}$ NMR $(600 \mathrm{MHz}) \delta 9.73(1 \mathrm{H}, \mathrm{s}, \mathrm{CHO})$, 7.60-7.36 (10H, m, $\mathrm{PhH}), 4.72,4.58\left(2 \mathrm{H}, 2 \times \mathrm{d}, J_{\mathrm{A}, \mathrm{B}}=6.5 \mathrm{~Hz}, \mathrm{CH}_{2} \mathrm{OCH}_{3}\right), 4.50\left(1 \mathrm{H}, \mathrm{d}, J_{2,3}=6.4 \mathrm{~Hz}\right.$, H-2), 4.44, $4.36\left(2 \mathrm{H}, 2 \times \mathrm{d}, J_{\mathrm{a}, \mathrm{b}}=9.6 \mathrm{~Hz}, \mathrm{H}^{\prime} 4^{\prime}\right), 4.40\left(1 \mathrm{H}, \mathrm{dd}, J_{3,5}=0.7 \mathrm{~Hz}, \mathrm{H}-3\right), 4.17\left(1 \mathrm{H}, \mathrm{dd}, J_{5,6}=2.1\right.$ Hz, H-5), 3.89 (1H, dd, $J_{6}{ }^{\prime}, 6=4.1 \mathrm{~Hz}, J_{6}{ }^{\prime}{ }^{\prime}, 6^{\prime} \mathrm{b}=10.8 \mathrm{~Hz}, \mathrm{H}-6$ ' a), 3.35 (1H, dd, $\left.J_{6 b^{\prime}, 6}=10.8 \mathrm{~Hz}, \mathrm{H}-6^{\prime} \mathrm{b}\right)$, $3.33\left(3 \mathrm{H}, \mathrm{s}, \mathrm{OCH}_{3}\right), 2.31(1 \mathrm{H}, \mathrm{ddd}, \mathrm{H}-6), 1.48,1.45,1.42,1.38\left(12 \mathrm{H}, 4 \times \mathrm{s}, 2 \times \mathrm{C}\left(\mathrm{CH}_{3}\right)_{2}\right), 1.03(9 \mathrm{H}, \mathrm{s}$, $\left.\mathrm{C}\left(\mathrm{CH}_{3}\right)_{3}\right)$. Anal. Calcd. for $\mathrm{C}_{33} \mathrm{H}_{45} \mathrm{O}_{8} \mathrm{~N}_{3} \mathrm{Si}$ (639.81): C, 61.95; H, 7.09; N, 6.57. Found: C, 61.85; H, 6.89; N, 6.97. 
1D-(1,2,3,4,5/3(OH),6)-6-Azido-5'-O-tert-butyldiphenylsilyl-6'-C-[2(S)-hydroxycyanomethyl]-3,5-di-

$C$-hydroxymethyl-1,2:3,3'-di- $O$-isopropylidene-4- $O$-methoxymethyl-1,2,3,4-cyclohexanetetrol (23),

and 1D-(1,2,3,4,5/3(OH),6)-6-Azido-5'-O-tert-butyldiphenylsilyl-6'-C-[2(R)-hydroxycyanomethyl]-3,

5-di- $C$-hydroxymethyl-1,2:3,3'-di- $O$-isopropylidene-4- $O$-methoxymethyl-1,2,3,4-cyclohexanetetrol

(23a). To a solution of aldehyde $22(570 \mathrm{mg}, 0.891 \mathrm{mmol})$ in $\mathrm{MeOH}(28 \mathrm{~mL})$, triethylamine $(2.22 \mathrm{~mL}$, $16.0 \mathrm{mmol})$ and trimethylsilyl cyanide $(2.34 \mathrm{~mL}, 17.8 \mathrm{mmol})$ were added, and stirred at $\mathrm{rt}$ for $6 \mathrm{~h}$. After the disappearance of $\mathbf{2 2}$ on TLC with 4:1 hexane-EtOAc, acetic acid ( $2 \mathrm{~mL}$ ) was added, and then the mixture was poured into brine, extracted with EtOAc, washed with brine, dried over anhyd $\mathrm{MgSO}_{4}$, and evaporated to give a residue. The remaining residue was purified on a column of silica gel with 4:1 hexane-EtOAc to give $23\left(R_{f} 0.3,331 \mathrm{mg}, 56 \%\right.$ yield $)$ and its epimer $23 \mathbf{a}\left(R_{f} 0.4,100 \mathrm{mg}, 17 \%\right.$ yield $)$, respectively.

Compound 23. mp $152.0-153.0{ }^{\circ} \mathrm{C}$ (colorless prisms, hexane-EtOAc); $[\alpha]_{\mathrm{D}}^{26}-0.60{ }^{\circ}\left(c 1.01, \mathrm{CHCl}_{3}\right)$; IR $\left(\mathrm{KBr}\right.$, disk) $v 3339 \mathrm{~cm}^{-1}(\mathrm{OH}), 2116 \mathrm{~cm}^{-1}\left(\mathrm{~N}_{3}\right)$; H NMR (600 MHz) $\delta 7.67-7.37(10 \mathrm{H}, \mathrm{m}, \mathrm{Ph} H), 5.18(1 \mathrm{H}$, $\left.\mathrm{d}, J_{6^{\prime}, \mathrm{OH}}=7.0 \mathrm{~Hz}, \mathrm{H}-6^{\prime}\right), 4.66,4.56\left(2 \mathrm{H}, 2 \times \mathrm{d}, J_{\mathrm{A}, \mathrm{B}}=6.7 \mathrm{~Hz}, \mathrm{CH}_{2} \mathrm{OCH}_{3}\right), 4.46\left(1 \mathrm{H}, \mathrm{d}, J_{1,2}=6.9 \mathrm{~Hz}, \mathrm{H}-1\right)$, $4.35(1 \mathrm{H}, \mathrm{d}, \mathrm{H}-2), 4.09\left(1 \mathrm{H}, \mathrm{dd}, J_{4,5}=3.4 \mathrm{~Hz}, \mathrm{H}-4\right), 3.88,3.81\left(2 \mathrm{H}, 2 \times \mathrm{d}, J_{\mathrm{a}, \mathrm{b}}=7.9 \mathrm{~Hz}, \mathrm{H}-3\right.$ '), $3.86(1 \mathrm{H}$, dd, $\left.J_{5^{\prime} \mathrm{a}, 5}=5.3 \mathrm{~Hz}, J_{5^{\prime} \mathrm{a}, 5^{\prime} \mathrm{b}}=8.9 \mathrm{~Hz}, \mathrm{H}-5^{\prime} \mathrm{a}\right), 3.35\left(1 \mathrm{H}, \mathrm{dd}, J_{5 \mathrm{~b}^{\prime}, 5}=8.9 \mathrm{~Hz}, \mathrm{H}-5^{\prime} \mathrm{b}\right), 3.68(1 \mathrm{H}, \mathrm{b} \quad \mathrm{d}, \mathrm{OH})$, $3.34\left(3 \mathrm{H}, \mathrm{s}, \mathrm{OCH}_{3}\right), 2.60(1 \mathrm{H}, \mathrm{ddd}, \mathrm{H}-5), 1.41,1.40,1.37,1.30\left(12 \mathrm{H}, 4 \times \mathrm{s}, 2 \times \mathrm{C}\left(\mathrm{CH}_{3}\right)_{2}\right), 1.09(9 \mathrm{H}, \mathrm{s}$, $\left.\mathrm{C}\left(\mathrm{CH}_{3}\right)_{3}\right)$. Anal. Calcd. for $\mathrm{C}_{34} \mathrm{H}_{46} \mathrm{O}_{8} \mathrm{~N}_{4} \mathrm{Si}$ (666.84): C, 61.24; H, 6.95; N, 8.40. Found: C, 61.22; H, 7.08; 
$\mathrm{N}, 8.24$.

Compound 23a. mp 132.0-133.0 ${ }^{\circ} \mathrm{C}$ (colorless prisms, hexane-EtOAc); $[\alpha]_{\mathrm{D}}^{26}+29.4^{\circ}\left(c 1.01, \mathrm{CHCl}_{3}\right)$; IR $(\mathrm{KBr}$, disk $) \vee 3447 \mathrm{~cm}^{-1}(\mathrm{OH}), 2116 \mathrm{~cm}^{-1}\left(\mathrm{~N}_{3}\right) . \quad$ H NMR $(600 \mathrm{MHz}) \delta 7.65-7.40(10 \mathrm{H}, \mathrm{m}, \mathrm{Ph} H), 5.28$ $\left(1 \mathrm{H}, \mathrm{d}, J_{6^{\prime}, \mathrm{OH}}=5.7 \mathrm{~Hz}, \mathrm{H}-6^{\prime}\right), 4.79,4.60\left(2 \mathrm{H}\right.$, each d, $\left.J_{\mathrm{A}, \mathrm{B}}=6.4 \mathrm{~Hz}, \mathrm{CH}_{2} \mathrm{OCH}_{3}\right), 4.29(1 \mathrm{H}, \mathrm{d}, \mathrm{OH}), 4.25$ $\left(1 \mathrm{H}, \mathrm{d}, J_{2,1}=6.7 \mathrm{~Hz}, \mathrm{H}-2\right), 4.09\left(1 \mathrm{H}, \mathrm{d}, J_{4,5}=4.6 \mathrm{~Hz}, \mathrm{H}-4\right), 4.05\left(1 \mathrm{H}, \mathrm{dd}, J_{1,5}=1.7 \mathrm{~Hz}, \mathrm{H}-1\right), 3.89(1 \mathrm{H}, \mathrm{dd}$, $J_{5^{\prime} \mathrm{a}, 5}=2.2 \mathrm{~Hz}, J_{5^{\prime}{ }^{\prime}, 5^{\prime} \mathrm{b}}=10.3 \mathrm{~Hz}, \mathrm{H}-5$ 'a $), 3.65\left(1 \mathrm{H}, \mathrm{dd}, J_{5 \mathrm{~b}^{\prime}, 5}=10.3 \mathrm{~Hz}, \mathrm{H}-5{ }^{\prime} \mathrm{b}\right), 3.54,3.49\left(2 \mathrm{H}\right.$, each d, $J_{\mathrm{a}, \mathrm{b}}$ $\left.=7.9 \mathrm{~Hz}, \mathrm{H}-3^{\prime}\right), 3.40\left(3 \mathrm{H}, \mathrm{s}, \mathrm{OCH}_{3}\right), 2.78(1 \mathrm{H}, \mathrm{dddd}, \mathrm{H}-5), 1.37,1.35,1.33,1.23(12 \mathrm{H}, 4 \times \mathrm{s}, 2$ $\left.\times \mathrm{C}\left(\mathrm{CH}_{3}\right)_{2}\right), 1.09\left(9 \mathrm{H}, \mathrm{s}, \mathrm{C}\left(\mathrm{CH}_{3}\right)_{3}\right)$. Anal. Calcd. for $\mathrm{C}_{34} \mathrm{H}_{46} \mathrm{O}_{8} \mathrm{~N}_{4} \mathrm{Si}(666.84): \mathrm{C}, 61.24 ; \mathrm{H}, 6.95 ; \mathrm{N}, 8.40$. Found: C, 61.11; H, 7.05; N, 8.33.

1D-(1,2,3,4,5/3(OH),6)-6-azido-5' -O-tert-butyldiphenylsilyl-6'- $C$-[2(S)-hydroxycyanomethyl]-3,5-di-

\section{$C$-hydroxymethyl-1,2:3,3'-di- $O$-isopropylidene-4,6'-di- $O$-methoxymethyl-1,2,3,4-cyclohexanetetrol}

(24). To a solution of cyanohydrin $23(17 \mathrm{mg}, 25 \mu \mathrm{mol})$ in $\mathrm{CH}_{2} \mathrm{Cl}_{2}(0.85 \mathrm{~mL})$, dimethoxymethane $(113 \mu \mathrm{L}$, $1.28 \mathrm{mmol})$ and Molecular Sieves $4 \mathrm{~A}(7 \mathrm{mg})$, and $\mathrm{P}_{2} \mathrm{O}_{5}(3 \mathrm{mg}, 20 \mu \mathrm{mol})$ were added, and stirred the solution at rt. After the disappearance of $\mathbf{2 3}$ on TLC with 4:1 hexane-EtOAc, the reaction mixture was quenched with triethylamine, then Molecular Sieves was filtered off, poured into aq $\mathrm{NaHCO}_{3}$ solution, extracted with $\mathrm{CHCl}_{3}$, washed with brine and water, dried over anhyd $\mathrm{MgSO}_{4}$, and evaporated to give a residue. The remaining residue was purified on a column of silica gel with 4:1 hexane-EtOAc to give $\mathbf{2 4}$ (17.1 mg, 94\% yield); mp $117.5-118.5{ }^{\circ} \mathrm{C}$ (colorless needles, hexane-EtOAc); $[\alpha]_{\mathrm{D}}^{26}-3.61{ }^{\circ}(c 0.95$, 
$\left.\mathrm{CHCl}_{3}\right) ; \mathrm{IR}\left(\mathrm{KBr}\right.$, Disk) v $2131 \mathrm{~cm}^{-1}\left(\mathrm{~N}_{3}\right) ; \quad \mathrm{H}$ NMR $(600 \mathrm{MHz}) \delta 7.68-7.36(10 \mathrm{H}, \mathrm{m}, \mathrm{Ph} H), 5.46(1 \mathrm{H}, \mathrm{s}$, H-6'), 4.94, $4.82\left(2 \mathrm{H}, 2 \times \mathrm{d}, J_{\mathrm{A}, \mathrm{B}}=6.7 \mathrm{~Hz}, \mathrm{CH}_{2} \mathrm{OCH}_{3}\right), 4.71,4.59\left(2 \mathrm{H}, 2 \times \mathrm{d}, J_{\mathrm{A}, \mathrm{B}}=6.5 \mathrm{~Hz}, \mathrm{CH}_{2} \mathrm{OCH}_{3}\right)$, $4.50\left(1 \mathrm{H}, \mathrm{d}, J_{4,5}=1.9 \mathrm{~Hz}, J_{4,2}=6.9 \mathrm{~Hz}, \mathrm{H}-4\right), 4.35(1 \mathrm{H}, \mathrm{dd}, \mathrm{H}-2), 4.22\left(1 \mathrm{H}, \mathrm{d}, J_{1,5}=4.5 \mathrm{~Hz}, \mathrm{H}-1\right), 3.71$ $\left(1 \mathrm{H}, \mathrm{dd}, J_{5}{ }^{\prime} \mathrm{a}, 5=2.8 \mathrm{~Hz}, J_{5}{ }^{\prime} \mathrm{a}, 5^{\prime} \mathrm{b}=10.5 \mathrm{~Hz}, \mathrm{H}-5{ }^{\prime} \mathrm{a}\right), 3.60,3.51\left(2 \mathrm{H}, 2 \times \mathrm{d}, J_{\mathrm{a}, \mathrm{b}}=7.7 \mathrm{~Hz}, \mathrm{H}-3^{\prime}\right), 3.57(1 \mathrm{H}, \mathrm{dd}$, $J_{5 \mathrm{~b}, 5}=10.8 \mathrm{~Hz}, \mathrm{H}-5$ 'b), 3.52, $3.36\left(6 \mathrm{H}, 2 \times \mathrm{s}, \mathrm{OCH}_{3}\right), 2.55(1 \mathrm{H}, \mathrm{dddd}, \mathrm{H}-5), 1.38,1.33,1.31,1.24(12 \mathrm{H}$, $\left.4 \times \mathrm{s}, 2 \times \mathrm{C}\left(\mathrm{CH}_{3}\right)_{2}\right), 1.10\left(9 \mathrm{H}, \mathrm{s}, \mathrm{C}\left(\mathrm{CH}_{3}\right)_{3}\right)$. Anal. Calcd. for $\mathrm{C}_{36} \mathrm{H}_{50} \mathrm{~N}_{4} \mathrm{O}_{9} \mathrm{Si}$ (710.89): C, 60.82; H, 7.09; $\mathrm{N}$, 7.88. Found: C, 60.79; H, 7.27; N, 7.58.

\section{D-(1,2,4,5/3(OH),6(N $\left.\left.\mathrm{N}_{3}\right)\right)$-6-Azido-5'-O-tert-butyldiphenylsilyl-1,2:3,3'-di-O-isopropylidene-4- $O$-met}

hoxymethyl-6- $C$-(S)-(6'-formyl-6'-methoxymethoxymethyl)-3,5-di- $C$-hydroxymethylcyclohexane-1,

2,3,4-tetrol (25). To a solution of $\mathbf{2 4}(239 \mathrm{mg}, 336 \mu \mathrm{mol})$ in toluene $(20 \mathrm{~mL})$, diisobutylaluminium hydride $(0.94 \mathrm{M}$ solution in hexane, $358 \mu \mathrm{L}, 336 \mu \mathrm{mol})$ was added dropwise, and stirred at $-78{ }^{\circ} \mathrm{C}$ under argon. After the disappearance of $\mathbf{2 4}$ on TLC with 4:1 hexane-EtOAc, acetic acid (300 $\mu \mathrm{L})$ was added to the reaction mixture, and then diluted with EtOAc $(20 \mathrm{~mL})$. Following, to the mixture, $1.0 \mathrm{M}$ aq $\mathrm{HCl}$ solution $(1.0 \mathrm{~mL})$ was also added and vigorously stirred for $10 \mathrm{~min}$. The organic layer was washed with aq $\mathrm{NaHCO}_{3}$ solution, brine, and water, dried over anhyd $\mathrm{MgSO}_{4}$, and evaporated to give 25 (209 $\mathrm{mg}, 87 \%$ yield), which was purified on a column of silica gel with 4:1 hexane-EtOAc; colorless syrup; $[\alpha]_{\mathrm{D}}^{26}$ $+49.3^{\circ}\left(c 1.21, \mathrm{CHCl}_{3}\right)$; IR (KBr, neat) $v 2108 \mathrm{~cm}^{-1}\left(\mathrm{~N}_{3}\right), 1738 \mathrm{~cm}^{-1}(\mathrm{C}=\mathrm{O}) ; \mathrm{H}$ NMR $(600 \mathrm{MHz}) \delta$ $10.17(1 \mathrm{H}, \mathrm{s}, \mathrm{CHO}), 7.67-7.36(10 \mathrm{H}, \mathrm{m}, \mathrm{PhH}), 5.29\left(1 \mathrm{H}, \mathrm{d}, J_{6}{ }^{\prime}, \mathrm{OH}=7.0 \mathrm{~Hz}, \mathrm{H}-6\right.$ ') $, 4.85,4.76(2 \mathrm{H}, 2 \times \mathrm{d}$, 
$\left.J_{\mathrm{A}, \mathrm{B}}=6.5 \mathrm{~Hz}, \mathrm{CH}_{2} \mathrm{OCH}_{3}\right), 4.71,4.59\left(2 \mathrm{H}, 2 \times \mathrm{d}, J_{\mathrm{A}, \mathrm{B}}=6.7 \mathrm{~Hz}, \mathrm{CH}_{2} \mathrm{OCH}_{3}\right), 4.55\left(1 \mathrm{H}, \mathrm{dd}, J_{1,2}=6.7 \mathrm{~Hz}\right.$, $\left.J_{1,5}=1.9 \mathrm{~Hz}, \mathrm{H}-1\right), 4.33(1 \mathrm{H}, \mathrm{d}, \mathrm{H}-2), 4.17(1 \mathrm{H}, \mathrm{d}, \mathrm{H}-4), 3.89\left(1 \mathrm{H}, \mathrm{dd}, J_{5}{ }^{\mathrm{a}, 5}=10.1 \mathrm{~Hz}, J_{5}{ }^{\prime} \mathrm{a}, 5^{\prime} \mathrm{b}=10.5 \mathrm{~Hz}\right.$, H-5'a), $3.35\left(1 \mathrm{H}, \mathrm{dd}, J_{5 \mathrm{~b}}{ }^{,}, 5=3.4 \mathrm{~Hz}, \mathrm{H}-5\right.$ 'b $), 3.67,3.59\left(2 \mathrm{H}, 2 \times \mathrm{d}, J_{\mathrm{a}, \mathrm{b}}=7.7 \mathrm{~Hz}, \mathrm{H}-3^{\prime}\right), 3.48,3.35(6 \mathrm{H}, 2$ $\left.\times \mathrm{s}, \mathrm{OCH}_{3}\right), 2.66(1 \mathrm{H}, \mathrm{dddd}, \mathrm{H}-5), 1.39,1.38,1.37,1.25\left(12 \mathrm{H}, 4 \times \mathrm{s}, 2 \times \mathrm{C}\left(\mathrm{CH}_{3}\right)_{2}\right), 1.03\left(9 \mathrm{H}, \mathrm{s}, \mathrm{C}\left(\mathrm{CH}_{3}\right)_{3}\right)$. Anal. Calcd. for $\mathrm{C}_{36} \mathrm{H}_{51} \mathrm{O}_{10} \mathrm{~N}_{3} \mathrm{Si}$ (713.89) : C, 60.57; H, 7.20; N, 5.89. Found: C, 60.58; H, 7.29; N, 6.16. 1D-(1,2,4,5/3(OH),6(N $\left.\left(\mathrm{N}_{3}\right)\right)$-6-Azido-5'-O-tert-butyldiphenylsilyl-1,2:3,3'-di- $O$-isopropylidene-6- $C$ - $(S)$-(

\section{6'-formyl-6'-methoxymethoxymethyl)-3,5-di- $C$-hydroxymethylcyclohexane-1,2,3,4-tetrol}

6",4-lactone (26). To a solution of $\mathbf{2 5}(73 \mathrm{mg}, 102 \mu \mathrm{mol})$ in 1:1 acetone- $\mathrm{CH}_{2} \mathrm{Cl}_{2}(8 \mathrm{~mL})$, Jones oxidizing reagent (2.67M aq $\mathrm{H}_{2} \mathrm{SO}_{4}$ solution, $90 \mu \mathrm{L}, 240 \mu \mathrm{mol}$ ) was added at $0{ }^{\circ} \mathrm{C}$, and stirred for $30 \mathrm{~min}$ at rt. First time, starting compound became a hemiacetal intermediate, and after which it gradually converted into the $\delta$-lactone 26. After the disappearance of this acetal on TLC with 3:1 hexane-EtOAc, the reaction mixture was quenched with isopropyl alcohol $(50 \mu \mathrm{L})$, poured into aq $\mathrm{NaHCO}_{3}$ solution, extracted with EtOAc, washed with brine and water, dried over anhyd $\mathrm{MgSO}_{4}$, and evaporated to give 26 (62 mg, $90 \%$ yield), which was purified on a column of silica gel with 4:1 hexane-EtOAc; colorless syrup; $[\alpha]_{\mathrm{D}}^{26}$ $-57.9^{\circ}\left(c 0.33, \mathrm{CHCl}_{3}\right) ; \mathrm{IR}(\mathrm{KBr}$, neat $) \vee 2108 \mathrm{~cm}^{-1}\left(\mathrm{~N}_{3}\right), 1738 \mathrm{~cm}^{-1}(\mathrm{C}=\mathrm{O}) ; \quad \mathrm{H}$ NMR $(600 \mathrm{MHz}) \delta$ 7.66-7.37 (10H, m, PhH), 4.81, $4.51\left(2 \mathrm{H}, 2 \times \mathrm{d}, J_{\mathrm{A}, \mathrm{B}} 6.5 \mathrm{~Hz}, \mathrm{CH}_{2} \mathrm{OCH}_{3}\right), 4.76(1 \mathrm{H}, \mathrm{s}, \mathrm{H}-4), 4.55(1 \mathrm{H}, \mathrm{d}$, $\left.J_{2,1}=6.4 \mathrm{~Hz}, \mathrm{H}-2\right), 4.43,4.27\left(2 \mathrm{H}, 2 \times \mathrm{d}, J_{\mathrm{a}, \mathrm{b}}=10.0 \mathrm{~Hz}, \mathrm{H}-3^{\prime}\right), 4.38\left(1 \mathrm{H}, \mathrm{d}, J_{6}, 5=0.9 \mathrm{~Hz}, \mathrm{H}-6^{\prime}\right), 4.31(1 \mathrm{H}$,

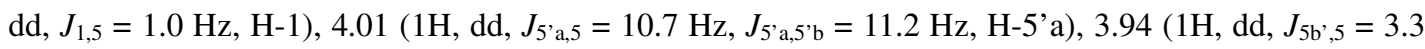


Hz, H-5’b), 3.27 (3H, s, $\left.\mathrm{OCH}_{3}\right), 2.41(1 \mathrm{H}, \mathrm{dddd}, \mathrm{H}-5), 1.52,1.46,1.45,1.37\left(12 \mathrm{H}, 4 \times \mathrm{s}, 2 \times \mathrm{C}\left(\mathrm{CH}_{3}\right)_{2}\right)$,

$1.05\left(9 \mathrm{H}, \mathrm{s}, \mathrm{C}\left(\mathrm{CH}_{3}\right)_{3}\right) ;{ }^{3} \mathrm{C} \mathrm{NMR}(150 \mathrm{MHz}) \delta 166.0,135.5,135.5,133.2,133.1,129.8,129.8,127.8$ $127.7,111.7,110.5,96.6,79.4,79.0,78.6,78.0,70.6,69.1,64.1,59.3,56.5,38.6,27.1,26.8,26.1,24.7$, 24.5, 19.2. Anal. Calcd. For $\mathrm{C}_{34} \mathrm{H}_{45} \mathrm{~N}_{3} \mathrm{O}_{9} \mathrm{Si}$ (667.29): C, 61.15; H, 6.79; N, 6.29. Found: C, 60.91; H, 6.52; N, 6.36.

Hemiacetal intermediate $\left(\mathbf{2 5}^{\prime}\right)$. colorless syrup; IR (KBr, neat) $v 3360 \mathrm{~cm}^{-1}(\mathrm{OH}), 2104 \mathrm{~cm}^{-1}\left(\mathrm{~N}_{3}\right) ;{ }^{1} \mathrm{H}$ NMR (600 MHz) $\delta 7.70-7.35(10 \mathrm{H}, \mathrm{m}, \mathrm{Ph} H), 5.04\left(1 \mathrm{H}, \mathrm{dd}, J_{7,5}=1.5 \mathrm{~Hz}, J_{7,8}=12.2 \mathrm{~Hz}, \mathrm{H}-6\right.$ ' $), 4.88(1 \mathrm{H}$, d, H-6"), 4.60, $4.51\left(2 \mathrm{H}, 2 \times \mathrm{d}, J_{\mathrm{A}, \mathrm{B}}=6.9 \mathrm{~Hz}, \mathrm{CH}_{2} \mathrm{OCH}_{3}\right), 4.52\left(1 \mathrm{H}, \mathrm{d}, J_{2,1}=8.9 \mathrm{~Hz}, \mathrm{H}-2\right), 4.49(1 \mathrm{H}, \mathrm{d}$, H-1), 4.38, $4.35\left(2 \mathrm{H}, 2 \times \mathrm{d}, J_{\mathrm{a}, \mathrm{b}}=9.6 \mathrm{~Hz}, \mathrm{H}^{\prime} 3^{\prime}\right), 4.30(1 \mathrm{H}, \mathrm{s}, \mathrm{H}-4), 4.10\left(1 \mathrm{H}, \mathrm{dd}, J_{5^{\prime} \mathrm{a}, 5}=10.1 \mathrm{~Hz}, J_{5^{\prime} \mathrm{a}, 5^{\prime} \mathrm{b}}=\right.$ $10.5 \mathrm{~Hz}, \mathrm{H}-5$ 'a), $3.83\left(1 \mathrm{H}, \mathrm{dd}, J_{5 \mathrm{~b}^{\prime}, 5}=2.8 \mathrm{~Hz}, \mathrm{H}-5{ }^{\prime} \mathrm{b}\right), 3.69(1 \mathrm{H}, \mathrm{s}, \mathrm{OH}), 3.20\left(3 \mathrm{H}, \mathrm{s}, \mathrm{OCH}_{3}\right), 1.97(1 \mathrm{H}$, dddd, $\mathrm{H}-5), 1.47,1.46,1.45,1.37\left(12 \mathrm{H}, 4 \times \mathrm{s}, 2 \times \mathrm{C}\left(\mathrm{CH}_{3}\right)_{2}\right), 1.03\left(9 \mathrm{H}, \mathrm{s}, \mathrm{C}\left(\mathrm{CH}_{3}\right)_{3}\right)$.

1D-(1,2,4,5/3(OH),6(N3))-6-Amino-5' - $O$-t-butyldiphenylsilyl-1,2:3,3'-di- $O$-isopropylidene-6-C-(S)-(6' -formyl-6'-methoxymethoxymethyl)-3,5-di-C-hydroxymethylcyclohexane-1,2,3,4-tetrol 6",4-lactone (27). $\delta$-Lactone 26 (95 mg, $0.142 \mathrm{mmol})$ was hydrogenated in $\mathrm{EtOH}(9.5 \mathrm{~mL})$ in the presence of $10 \%$ $\mathrm{Pd}-\mathrm{C}$ (30 mg) under hydrogen for $7 \mathrm{~h}$. After the disappearance of $\mathbf{2 6}$ on TLC with 2:1 hexane-EtOAc, the catalyst was filtered off, and evaporated to give 27 (91 mg, quantitatively), which was purified on a column of silica gel with 30:15:2 hexane-EtOAc-Et $3 \mathrm{~N}$; colorless syrup; $[\alpha]_{\mathrm{D}}^{26}-53.5^{\circ}\left(c 0.73, \mathrm{CHCl}_{3}\right)$; IR $(\mathrm{KBr}$, neat $) \vee 3385 \mathrm{~cm}^{-1}\left(\mathrm{NH}_{2}\right), v 1771 \mathrm{~cm}^{-1}(\mathrm{C}=\mathrm{O}) ; \quad \mathrm{H}$ NMR $(600 \mathrm{MHz}) \delta 7.69-7.37(10 \mathrm{H}, \mathrm{m}, \mathrm{Ph} H)$, 
$4.88,4.50\left(2 \mathrm{H}, 2 \times \mathrm{d}, J_{\mathrm{A}, \mathrm{B}}=6.4 \mathrm{~Hz}, \mathrm{CH}_{2} \mathrm{OCH}_{3}\right), 4.72\left(1 \mathrm{H}, \mathrm{dd}, J_{4,2}=1.4 \mathrm{~Hz}, J_{4,5}=1.0 \mathrm{~Hz}, \mathrm{H}-4\right), 4.40$, $4.28\left(2 \mathrm{H}, 2 \times \mathrm{d}, J_{\mathrm{a}, \mathrm{b}}=9.8 \mathrm{~Hz}, \mathrm{H}-3\right.$ ') $, 4.26\left(1 \mathrm{H}, \mathrm{dd}, J_{2,1}=6.2 \mathrm{~Hz}, J_{2,4}=1.2 \mathrm{~Hz}, \mathrm{H}-2\right), 4.18(1 \mathrm{H}, \mathrm{d}, \mathrm{H}-5)$, $4.09\left(1 \mathrm{H}, \mathrm{d}, J_{6}{ }^{\prime}, 5=1.2 \mathrm{~Hz}, \mathrm{H}-6^{\prime}\right), 3.94\left(1 \mathrm{H}, \mathrm{dd}, J_{5^{\prime} \mathrm{a}, 5}=10.5 \mathrm{~Hz}, J_{5}{ }^{\prime} \mathrm{a}, 5^{\prime} \mathrm{b}=10.7 \mathrm{~Hz}, \mathrm{H}-5^{\prime} \mathrm{a}\right), 3.83(1 \mathrm{H}, \mathrm{dd}$, $J_{5 \mathrm{~b}^{\prime}, 5}=3.8 \mathrm{~Hz}, \mathrm{H}-5$ 'b), $3.23\left(3 \mathrm{H}, \mathrm{s}, \mathrm{OCH}_{3}\right), 2.27\left(1 \mathrm{H}\right.$, dddd, H-5), $1.52\left(2 \mathrm{H}\right.$, br s, $\left.\mathrm{NH}_{2}\right), 1.50,1.46,1.45$, $1.34\left(12 \mathrm{H}, 4 \times \mathrm{s}, 2 \times \mathrm{C}\left(\mathrm{CH}_{3}\right)_{2}\right), 1.04\left(9 \mathrm{H}, \mathrm{s}, \mathrm{C}\left(\mathrm{CH}_{3}\right)_{3}\right) ; \quad{ }^{3} \mathrm{C} \mathrm{NMR}(150 \mathrm{MHz}) \delta 165.7,135.6,135.5,132.5$, $132.4,129.9,127.9,127.8,111.7,110.5,97.3,78.4,77.6,77.5,77.2,71.2,68.9,60.3,58.3,57.2,27.0$, 26.9, 26.8, 26.7, 26.0, 24.9, 24.5, 19.1, 18.4. nal. Calcd. for $\mathrm{C}_{34} \mathrm{H}_{47} \mathrm{NO}_{9} \mathrm{Si}$ (641.82): C, 63.63; H, 7.38; N, 2.18. Found: C, 63.86; H, 7.17; N, 2.18 .

1D-(1,2,4,5/3(OH),6(NH2))-6-Amino-1,2:3,3'-di- $O$-isopropylidene-6- $C$-(S)-6'-methoxymethoxymethy l-3,5-di-C-hydroxymethyl-cyclohexane-1,2,3,4-tetrol-6",4-lactone (28). To a solution of 27 (14.1 mg, $22 \mu \mathrm{mol})$ in THF ( $2 \mathrm{~mL})$, tetra- $n$-butylammonium fluoride (1.0M solution in THF, $33 \mu \mathrm{L}, 33 \mu \mathrm{mol})$ was added, and stirred for 30 min at rt. After the disappearance of 27 on TLC with 1:2 hexane-EtOAc, the reaction mixture was evaporated to give a residue. The remaining residue was purified on a column of silica gel with 50:50:2 hexane-EtOAc-Et ${ }_{3} \mathrm{~N}$ to give $\mathbf{2 8}\left(8.0 \mathrm{mg}, 90 \%\right.$ yield); colorless syrup; $[\alpha]_{\mathrm{D}}^{25}$ $+25.0^{\circ}\left(c 0.3, \mathrm{CHCl}_{3}\right)$; IR (KBr, neat) $v 3500 \mathrm{~cm}^{-1}(\mathrm{OH}), 3500 \mathrm{~cm}^{-1}\left(\mathrm{NH}_{2}\right), 1771 \mathrm{~cm}^{-1}(\mathrm{C}=\mathrm{O}) ; \quad \mathrm{H}$ NMR $(600 \mathrm{MHz}) \delta 5.00,4.82\left(2 \mathrm{H}, 2 \times \mathrm{d}, J_{\mathrm{A}, \mathrm{B}}=7.0 \mathrm{~Hz}, \mathrm{CH}_{2} \mathrm{OCH}_{3}\right), 4.74(1 \mathrm{H}, \mathrm{s}, \mathrm{H}-6$ ' $), 4.54\left(1 \mathrm{H}, \mathrm{d}, J_{1,2}=6.9\right.$ $\mathrm{Hz}, \mathrm{H}-1), 4.43\left(1 \mathrm{H}, \mathrm{dd}, J_{5}{ }^{\prime} \mathrm{a}, 5=12.0 \mathrm{~Hz}, J_{5}{ }^{\prime}, 5^{\prime} \mathrm{b}=12.2 \mathrm{~Hz}, \mathrm{H}-5{ }^{\prime} \mathrm{a}\right), 4.42\left(1 \mathrm{H}, \mathrm{d}, J_{2,4}=1.4 \mathrm{~Hz}, \mathrm{H}-2\right), 4.35$ $\left(1 \mathrm{H}, \mathrm{dd}, J_{5 \mathrm{~b}^{\prime}, 5}=5.3 \mathrm{~Hz}, \mathrm{H}-5\right.$ 'b), 4.33, $4.23\left(2 \mathrm{H}, 2 \times \mathrm{d}, J_{\mathrm{a}, \mathrm{b}}=9.6 \mathrm{~Hz}, \mathrm{H}-3^{\prime}\right), 3.78\left(1 \mathrm{H}, \mathrm{ddd}, J_{4,5}=5.5 \mathrm{~Hz}\right.$, 
$\left.J_{4, \mathrm{OH}}=12.2 \mathrm{~Hz}, \mathrm{H}-4\right), 3.48\left(3 \mathrm{H}, \mathrm{s}, \mathrm{OCH}_{3}\right), 2.96\left(1 \mathrm{H}, \mathrm{d}, J_{4, \mathrm{OH}}=12.4 \mathrm{~Hz}, \mathrm{OH}\right), 2.60(1 \mathrm{H}, \mathrm{ddd}, \mathrm{H}-5), 1.87$

$\left(2 \mathrm{H}\right.$, br s, $\left.\mathrm{NH}_{2}\right), 1.48,1.41,1.40,1.39\left(12 \mathrm{H}, 4 \times \mathrm{s}, 2 \times \mathrm{C}\left(\mathrm{CH}_{3}\right)_{2}\right)$. Anal. Calcd. for $\mathrm{C}_{18} \mathrm{H}_{29} \mathrm{NO}_{9}(403.42): \mathrm{C}$,

53.59; H, 7.25; N, 3.47. Found: C, 53.98; H, 7.22; N, 3.09.

1D-(1,2,4,5/3(OH),6(NH))-6- $N$-[N,N'-bis-(tert-buthoxycarbonyl)-guanidino]-1,2:3,3'-di- $O$-isopropyli

dene-6-C-(S)-(6'-methoxymethoxymethyl)-3,5-di-C-hydroxymethylcyclohexane-1,2,3,4-tetrol-6",4-1

actone (29). To a solution of amine $28(20 \mathrm{mg}, 49.6 \mu \mathrm{mol})$ in dry $N, N^{\prime}$-dimethylformamide $(2 \mathrm{~mL})$, $N, N$ '-bis(tert-butoxycarbonyl)thiourea (15 mg, $51.6 \mu \mathrm{mol})$, mercury(II) chloride (14 mg, $51.6 \mu \mathrm{mol})$, and triethylamine $(10 \mu \mathrm{L}, 72.3 \mu \mathrm{mol})$ were added and stirred at $\mathrm{rt}$. After $10 \mathrm{~min}$, to the reaction mixture, $N, N$ '-bis(tert-butoxycarbonyl)thiourea (15 mg, $51.6 \mu \mathrm{mol})$, mercury(II) chloride (14 mg, $51.6 \mu \mathrm{mol})$, and triethylamine $(10 \mu \mathrm{L}, 72.3 \mu \mathrm{mol})$ were added again, and stirred at $\mathrm{rt}$ for $10 \mathrm{~min}$. Above procedure was repeated another three times with the use of $N, N^{\prime}$-bis(tert-butoxycarbonyl) thiourea $(15 \mathrm{mg}, 51.6 \mu \mathrm{mol} \times$ 3), mercury(II) chloride (14 mg, $51.6 \mu \mathrm{mol} \times 3)$, and triethylamine $(10 \mu \mathrm{L}, 72.3 \mu \mathrm{mol} \times 3)$. After the disappearance of $\mathbf{2 8}$ on TLC with 1:1 hexane-EtOAc, the reaction mixture was filtered through a pad of celite, washed with chloroform, evaporated to give a residue. The remaining residue was purified on a column of silica gel with 5:1 hexane-EtOAc to give guanidine derivative $\mathbf{2 9}(24.7 \mathrm{mg}, 77 \%$ yield). This compound exist a mixture of conformational isomers in chloroform solution (major:minor 3:1); colorless syrup; $[\alpha]_{\mathrm{D}}^{25}+1.54^{\circ}\left(c 0.86, \mathrm{CDCl}_{3}\right)$; IR (KBr neat) $v 3527 \mathrm{~cm}^{-1}(\mathrm{OH}), 3257 \mathrm{~cm}^{-1}(\mathrm{NH}), 1747,1726$, $1645,1633,1626 \mathrm{~cm}^{-1}(\mathrm{C}=\mathrm{O}$ and $\mathrm{C}=\mathrm{N}) ;{ }^{1} \mathrm{H} \mathrm{NMR}(600 \mathrm{MHz})$ major conformational isomer $\delta 11.29(1 \mathrm{H}$, 
s, NH), 8,49 (1H, s, NH), $5.18\left(1 \mathrm{H}, \mathrm{s}, \mathrm{H}-6^{\prime}\right), 5.13\left(1 \mathrm{H}, \mathrm{d}, J_{1,2}=6.9 \mathrm{~Hz}, \mathrm{H}-1\right), 4.89,4.84\left(2 \mathrm{H}, 2 \times \mathrm{d}, J_{\mathrm{A}, \mathrm{B}}=\right.$ $\left.6.4 \mathrm{~Hz}, \mathrm{CH}_{2} \mathrm{OCH}_{3}\right), 4.73\left(1 \mathrm{H}, \mathrm{dd}, J_{5^{\prime} \mathrm{a}, 5^{\prime} \mathrm{b}}=5.5 \mathrm{~Hz}, J_{5}{ }^{\prime} \mathrm{a}, 5=11.9 \mathrm{~Hz}, \mathrm{H}-5{ }^{\prime} \mathrm{a}\right), 4.36\left(1 \mathrm{H}, \mathrm{dd}, J_{2,4}=1.0 \mathrm{~Hz}\right.$, $\mathrm{H}-2), 4.36\left(1 \mathrm{H}, \mathrm{dd}, J_{5}{ }^{\prime} \mathrm{b}, 5=5.5 \mathrm{~Hz}, \mathrm{H}-5^{\prime} \mathrm{b}\right), 4.32,4.22\left(2 \mathrm{H}, 2 \times \mathrm{d}, J_{\mathrm{a}, \mathrm{b}}=9.6 \mathrm{~Hz}, \mathrm{H}-3^{\prime}\right), 3.83\left(1 \mathrm{H}, \mathrm{ddd}, J_{4,5}=\right.$ $\left.6.2 \mathrm{~Hz}, J_{4, \mathrm{OH}}=11.7 \mathrm{~Hz}, \mathrm{H}-4\right), 3.41\left(3 \mathrm{H}, \mathrm{s}, \mathrm{CH}_{2} \mathrm{OCH}_{3}\right), 3.08(1 \mathrm{H}, \mathrm{ddd}, \mathrm{H}-5), 2.71(1 \mathrm{H}, \mathrm{d}, \mathrm{OH}), 1.48,1.47$ $\left(18 \mathrm{H}, 2 \times \mathrm{s}, 2 \times \mathrm{COC}\left(\mathrm{CH}_{3}\right)_{3}\right), 1.48,1.47,1.39,1.32\left(12 \mathrm{H}, 4 \times \mathrm{s}, 2 \times \mathrm{C}\left(\mathrm{CH}_{3}\right)_{2}\right) ;$ minor conformational isomer $\delta 11.32(1 \mathrm{H}, \mathrm{s}, \mathrm{NH}), 8,80(1 \mathrm{H}, \mathrm{s}, \mathrm{NH}), 5.61\left(1 \mathrm{H}, \mathrm{s}, J_{6}, 5=6.4 \mathrm{~Hz}, \mathrm{H}-6{ }^{\prime}\right), 5.11,4.79\left(2 \mathrm{H}, 2 \times \mathrm{d}, J_{\mathrm{a}, \mathrm{b}}\right.$ $\left.=6.2 \mathrm{~Hz}, \mathrm{CH}_{2} \mathrm{OCH}_{3}\right), 4.66\left(1 \mathrm{H}\right.$, br s, H-2), $4.47(1 \mathrm{H}, \mathrm{s}, \mathrm{H}-1), 4.42,4.24\left(2 \mathrm{H}, 2 \times \mathrm{d}, J_{\mathrm{a}, \mathrm{b}}=9.8 \mathrm{~Hz}, \mathrm{H}-3^{\prime}\right)$, $3.82\left(1 \mathrm{H}\right.$, br dddd, $\left.J_{5,5^{\prime} \mathrm{a}}=2.4 \mathrm{~Hz}, J_{5,5^{\prime} \mathrm{b}}=7.4 \mathrm{~Hz}, \mathrm{H}-5\right), 4.36\left(1 \mathrm{H}, \mathrm{d}, J_{4,5}=3.4 \mathrm{~Hz}, \mathrm{H}-4\right), 3.79(1 \mathrm{H}, \mathrm{dd}$, H-5’b), $3.48\left(3 \mathrm{H}, \mathrm{s}, \mathrm{CH}_{2} \mathrm{OCH}_{3}\right), 2.92(1 \mathrm{H}, \mathrm{s}, \mathrm{H}-\mathrm{OH}), 1.48,1.47\left(18 \mathrm{H}, 2 \times \mathrm{s}, 2 \times \mathrm{COC}\left(\mathrm{CH}_{3}\right)_{3}\right), 1.54,1.46$, 1.44, $1.37\left(12 \mathrm{H}, 4 \times \mathrm{s}, 2 \times \mathrm{C}\left(\mathrm{CH}_{3}\right)_{2}\right) ; \quad{ }^{3} \mathrm{C} \mathrm{NMR}(150 \mathrm{MHz}) \delta 168.6,165.8,162.6,162.4,154.3,153.5$, $152.9,152.8,148.6,111.3,111.2,109.6,109.5,97.4,97.1,83.3,83.0,79.1,79.0,78.6,78.5,74.7,74.0$, $73.1,71.3,70.3,69.2,69.1,65.8,59.6,59.4,57.6,57.2,56.6,39.1,35.5,28.3,28.1,28.0,27.2,27.0,26.2$, 25.9, 25.2 24.9, 24.5, 23.5. ESI-TOF-MS Calcd for $\mathrm{C}_{29} \mathrm{H}_{48} \mathrm{~N}_{3} \mathrm{O}_{13} \mathrm{~m} / z[\mathrm{M}+\mathrm{H}]^{+}$: 646.3182. Found: 646.3187.

(-)-4,9-Anhydro-epi-TTX (1a). To a solution of $N, N^{\prime}$ '-bis(tert-butoxycarbonyl)guanidine 29 (8.6 mg, 152 $\mu \mathrm{mol})$ in dry dichloromethane $(0.4 \mathrm{~mL})$, pyridinium chlorochromate (PCC) (33 $\mathrm{mg}, 152 \mu \mathrm{mol})$ and celite (10 $\mathrm{mg})$ were added, and stirred at $\mathrm{rt}$ for $9 \mathrm{~h}$. After the disappearance of $\mathbf{2 9}$ on TLC with 1:1 hexane-EtOAc and 10:1 $\mathrm{CHCl}_{3}-\mathrm{MeOH}$, the reaction mixture was filtered through a pad of celite, washed 
with chloroform, evaporated to give a residue. The remaining residue was purified on a column of silica gel with EtOAc to give oxidation mixture quantitatively. Following, the mixture was dissolved in $\mathrm{MeOH}$ $(0.4 \mathrm{~mL})$ and $\mathrm{HCl}(4 \mathrm{M}$ solution of 1,4-dioxane, $0.4 \mathrm{~mL})$, and stirred at $\mathrm{rt}$ for $3 \mathrm{~h}$. The mixture was concentrated under reduced pressure to give a residue. The remaining residue was dissolved in $30 \%$ aq TFA, and stirred at $\mathrm{rt}$ for $6 \mathrm{~h}$. After the formation of anhydro-epi-TTX on TLC with 15:9:3:6 pyridine-EtOAc- $\mathrm{AcOH}-\mathrm{H}_{2} \mathrm{O}$, the mixture was concentrated under reduced pressure to give a residue. The residue was first purified by gel-column Sephadex G-15 $(17 \times 500 \mathrm{~mm}, 0.05 \mathrm{~N}$ aq AcOH$)$, and following by HPLC on a Hitachi-gel \#3013-c column $\left(\mathrm{H}^{+}\right.$form, $4.6 \times 150 \mathrm{~mm}, 0.05 \mathrm{~N}$ aq AcOH, peaks were monitored by $\left.\mathrm{RID}^{15 \mathrm{a}}\right)$ to give (-)-anhydro-epi-TTX (1.3 $\mathrm{mg}, 50 \%$ yield), and a small amount of TTX, respectively; white powder; $[\alpha]_{\mathrm{D}}^{28}+1.2^{\circ}(c 0.18,3 \% \mathrm{AcOH}) ;{ }^{1} \mathrm{H} \mathrm{NMR}(600 \mathrm{MHz}$, in $3 \%$ $\mathrm{CD}_{3} \mathrm{COOD} / \mathrm{D}_{2} \mathrm{O}$, referenced to $\left.\mathrm{CHD}_{2} \mathrm{COOD}(2.06 \mathrm{ppm})\right) \delta 5.53(1 \mathrm{H}, \mathrm{s}, \mathrm{H}-4), 4.63(1 \mathrm{H}, \mathrm{d}, J=2.1 \mathrm{~Hz}$, H-8), $4.58(1 \mathrm{H}, \mathrm{s}, \mathrm{H}-9), 4.36(1 \mathrm{H}, \mathrm{dd}, J=2.2 \mathrm{~Hz}, J=2.6 \mathrm{~Hz}, \mathrm{H}-5), 4.17(1 \mathrm{H}, \mathrm{t}, J=2.1 \mathrm{~Hz}, J=2.6 \mathrm{~Hz}$, H-7), 4.00, $3.92(2 \mathrm{H}, 2 \times \mathrm{d}, J=12.2 \mathrm{~Hz}, \mathrm{H}-11), 2.94(1 \mathrm{H}, \mathrm{d}, J=2.6 \mathrm{~Hz}, \mathrm{H}-4 \mathrm{a})$. ESI-TOF-MS Calcd for $\mathrm{C}_{11} \mathrm{H}_{16} \mathrm{~N}_{3} \mathrm{O}_{7} \mathrm{~m} / z,[\mathrm{M}+\mathrm{H}]^{+}:$302.0983. Found: 302.1022.

(-)-Tetrodotoxin (1). (-)-anhydro-epi-TTX $(2.1 \mathrm{mg}, 6.95 \mu \mathrm{mol})$ was dissolved in $4 \%$ AcOH $(0.4 \mathrm{~mL})$ (ca 3:1 mixture of TTX and anhydro-epi-TTX by NMR). The residue was purified by HPLC on a Hitachi-gel \#3013-c column ( $\mathrm{H}^{+}$form, $4.6 \times 150$ mm, $0.05 \mathrm{~N}$ aq AcOH, peaks were monitored by RID ${ }^{15 a}$ ) 
to give (-)-TTX (1.25 mg, including a slight lactone form in ${ }^{1} \mathrm{H}$ NMR), and recovered

(-)-anhydro-epi-TTX (0.4 mg), respectively; white powder; $[\alpha]_{\mathrm{D}_{\mathrm{A}}}^{28}-3.75^{\circ}(c 0.13,3 \% \mathrm{AcOH}) ;{ }^{1} \mathrm{H}$ NMR

(600 MHz, in $3 \% \mathrm{CD}_{3} \mathrm{COOD} / \mathrm{D}_{2} \mathrm{O}$, referenced to $\left.\mathrm{CHD}_{2} \mathrm{COOD}(2.06 \mathrm{ppm})\right) \delta 5.50(1 \mathrm{H}, \mathrm{d}, J=8.9 \mathrm{~Hz}$,

H-4), $4.30(1 \mathrm{H}, \mathrm{d}, J=2.1 \mathrm{~Hz}, \mathrm{H}-8), 4.25(1 \mathrm{H}, \mathrm{br} \mathrm{s}, \mathrm{H}-5), 4.09(1 \mathrm{H}, \mathrm{t}, J=2.1 \mathrm{~Hz}, \mathrm{H}-7), 4.06,4.01(2 \mathrm{H}, 2 \times$

$\mathrm{d}, J=12.4 \mathrm{~Hz}, \mathrm{H}-11), 3.96(1 \mathrm{H}, \mathrm{s}, \mathrm{H}-9), 2.35(1 \mathrm{H}, \mathrm{d}, J=9.6 \mathrm{~Hz}, \mathrm{H}-4 \mathrm{a})$. ESI-TOF-MS Calcd for

Formatted: Font: $13 \mathrm{pt}$

$\mathrm{C}_{11} \mathrm{H}_{18} \mathrm{~N}_{3} \mathrm{O}_{8} \mathrm{~m} / z,[\mathrm{M}+\mathrm{H}]^{+}: 320.1094$. Found: 320.1085 .

FIGURE. ${ }^{1} \mathrm{H}$ NMR spectra of tetrodotoxin after purification.

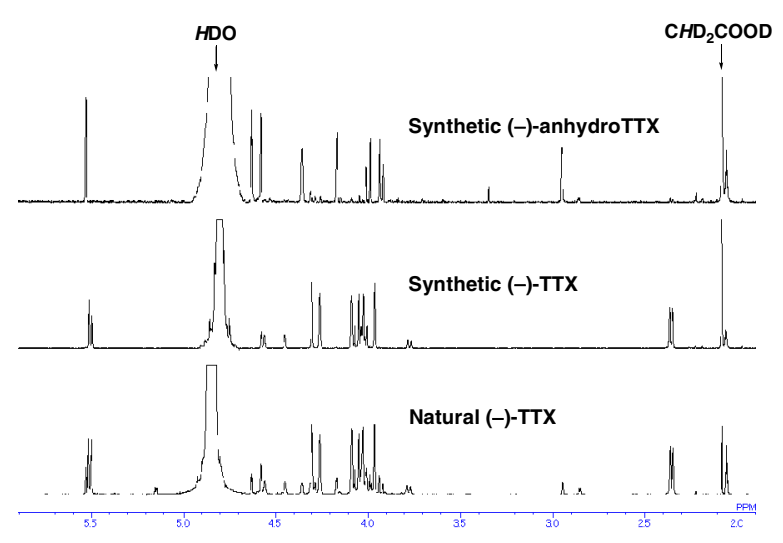

